\title{
Nonalcoholic Fatty Liver Disease: Pathologic Patterns and Biopsy Evaluation in Clinical Research
}

\author{
David E. Kleiner, M.D., Ph.D. ${ }^{1}$ Elizabeth M. Brunt, M.D. ${ }^{2}$
}

${ }^{1}$ Laboratory of Pathology, National Cancer Institute, National Institutes of Health, Bethesda, Maryland

2 Department of Pathology and Immunology, Washington University School of Medicine, St. Louis, Missouri
Address for correspondence and reprint requests David E. Kleiner, M.D., Ph.D., Laboratory of Pathology/National Cancer Institute, 10 Center Drive, Building 10, Room 2B50, MSC 1500, Bethesda, MD 20892 (e-mail: kleinerd@mail.nih.gov).

Semin Liver Dis 2012;32:3-13.

\begin{abstract}
Keywords

- Bariatric surgery

- disease classification

- lipodystrophy

- liver biopsy

- nonalcoholic fatty liver disease

- pediatric fatty liver disease

- steatohepatitis

- steatosis

Nonalcoholic fatty liver disease (NAFLD) results in histologically complex specific and nonspecific injury patterns. In clinical research of NAFLD, the liver biopsy evaluation provides a wealth of information on the architectural arrangement and severity of a variety of histologic changes, including steatosis, inflammation, cellular injury, and fibrosis. This information is summarized as an overall diagnostic category, such steatosis or steatohepatitis and the severity of the injury can be graded and staged. Histopathologic disease classification in NAFLD is related to but separate from evaluation of individual histologic lesions. The patient population under study may affect the prevalence of histologic findings and in particular, pediatric patients with NAFLD may show a higher prevalence of zone 1 steatosis and periportal fibrosis as compared with adult populations. For the purposes of clinical research, it is important to provide the pathologist with biopsies that are adequate to classify the disease process as well as to grade and stage the changes. A current understanding of NAFLD pathologic classification, as well as nuances of grading and staging, is presented in this review.
\end{abstract}

\section{Definition and Classification of Fatty Liver Disease}

If one examines the introductory text from almost any article written about nonalcoholic fatty liver disease (NAFLD) or nonalcoholic steatohepatitis (NASH), one is almost certain to find a phrase that describes NAFLD as both "identical to alcoholic liver disease" and "a spectrum of changes from steatosis to NASH to cirrhosis." Although there is some truth to these phrases, both are problematic. Although there may be significant overlap with features of alcoholic liver disease, there are also distinctions between the two. ${ }^{1}$ Second, the concept of the spectrum oversimplifies a complex disease, and implies that there is a continuous and unbroken movement from steatosis on one end to cirrhosis on the other. Further, it suggests a disease categorization that is one- dimensional and limited to a few disease states. The natural history of NAFLD on an individual patient level is largely unknown and it is not clear that all patients with steatosis are at risk for steatohepatitis or advanced fibrosis. Patients with steatohepatitis may cycle in and out of states in which that diagnosis can be recognized histologically, depending on therapeutic interventions and/or lifestyle modifications. There may be more than one path to cirrhosis depending on a variety of factors including the age of the patient. Disease classification in NAFLD must be robust enough to account for these possibilities.

In addition to these disease classification and natural history issues, NAFLD itself is a histologically complex disease. Unlike chronic hepatitis, which can be adequately graded and staged by characterizing the inflammation and the fibrosis, the histologic characterization of NAFLD and
Issue Theme NAFLD: Old Issues and Emerging Concepts; Guest Editor, Giovanni Musso, M.D.
Copyright @ 2012 by Thieme Medical Publishers, Inc., 333 Seventh Avenue, New York, NY 10001, USA. Tel: +1(212) 584-4662.
DOI http://dx.doi.org/ 10.1055/s-0032-1306421. ISSN 0272-8087. 
4 Nonalcoholic Fatty Liver Disease Kleiner, Brunt

Table 1 Histologic Categorization of Disease States in Patients at Risk for Nonalcoholic Fatty Liver Disease (NAFLD)

\begin{tabular}{|l|l|}
\hline Category & Definition \\
\hline $\begin{array}{l}\text { No significant evidence of fatty liver } \\
\text { disease (Not NAFLD) }\end{array}$ & $\begin{array}{l}\text { Insufficient steatosis for a diagnosis of steatosis (the NASH CRN uses a } \\
\text { threshold of 5\% of hepatocytes showing steatosis), without other } \\
\text { changes (ballooning, fibrosis) that would suggest steatohepatitis. }\end{array}$ \\
\hline $\begin{array}{l}\text { Steatosis } \\
\text { - Steatosis with inflammation }\end{array}$ & $\begin{array}{l}\text { Steatosis without specific changes to suggest a form of steatohepatitis. } \\
\text { This category may include spotty lobular inflammation and/or mild } \\
\text { degrees of fibrosis of uncertain significance. }\end{array}$ \\
\hline $\begin{array}{l}\text { Steatohepatitis } \\
\text { Z Zone } 3 \text { borderline steatohepatitis }\end{array}$ & $\begin{array}{l}\text { Form of steatohepatitis most common in adults; defined as a zone } \\
\text { 3 centered injury pattern that includes steatosis, inflammation, ballooning } \\
\text { injury (often with Mallory-Denk bodies) with or without fibrosis. Borderline } \\
\text { steatohepatitis has some, but not all of the features that would allow a } \\
\text { diagnosis of steatohepatitis. }\end{array}$ \\
\hline Zone 1, Borderline pattern & $\begin{array}{l}\text { Form of steatohepatitis that occurs mainly in young children, characterized by } \\
\text { zone 1-centered injury (portal inflammation, portal-based fibrosis, zone } \\
1 \text { steatosis, ballooning injury in zone 1 if present). }\end{array}$ \\
\hline Cryptogenic fibrosis/cirrhosis & $\begin{array}{l}\text { Presence of fibrosis (usually advanced) or cirrhosis, with little to no steatosis } \\
\text { and no changes (ballooning, Mallory-Denk bodies) that would suggest } \\
\text { borderline or definite steatohepatitis. Other explanations for fibrosis } \\
\text { (besides steatohepatitis) should be considered. }\end{array}$ \\
\hline
\end{tabular}

NASH CRN, Non-alcoholic Steatohepatitis Clinical Research Network.

NASH by definition includes description of steatosis and cell injury in addition to inflammation and fibrosis. These histologic features are not specific by themselves and may be found as components in other liver diseases. When they combine in specific patterns, we can recognize specific disease states such as steatohepatitis, even in conjunction with other diseases. At times, however, the pathologist may be limited to describing the degree of necroinflammatory injury and carefully cataloguing the changes seen. We propose that, for the purposes of clinical investigation, pathologists and clinicians categorize the histologic changes from biopsies done to evaluate NAFLD into sets of diagnoses as outlined in -Tables $\mathbf{1}$ and $\mathbf{2}$ and described in detail below. Because many of the individual histologic features of NAFLD may occur in other liver diseases and there remains no positive serologic test for NAFLD, there should be adequate clinical exclusion of other forms of liver diseases prior to categorizing a biopsy as a type of NAFLD.

\section{No Significant Evidence of Fatty Liver Disease (Not NAFLD)}

When patients with suspected fatty liver disease are biopsied, one possible outcome is that there is no evidence of fatty liver disease. This is known to occur in up to $10 \%$ of bariatric biopsies and has been reported in nonmorbidly obese individuals as well. ${ }^{2-4}$ In addition, it might be the result of effective therapy or might simply be the patient's true baseline state. Minimal steatosis might be present and for the purposes of clinical scientific study, the NASH CRN (Nonalcoholic Steatohepatitis Clinical Research Network) has used a literature based cutoff of $5 \%$ steatosis (meaning that less than $5 \%$ of hepatocytes show steatosis) as the threshold. ${ }^{5,6}$ Other mild, but nonspecific changes (including minimal inflammation or fibrosis) could be present without affecting the categorization. These findings may represent prior injury and resolution and may warrant a descriptive diagnosis and comment in the pathology report. Diffuse glycogenosis might be present and may provide an explanation for imaging findings. In routine clinical practice in which patients are screened by current generation imaging techniques prior to biopsy, this diagnosis should be unusual. In the setting of clinical research, patients who are at risk for NAFLD, but have no histologic evidence of disease would make an excellent control group for understanding the pathophysiology of steatosis.

\section{Steatosis}

It is important to remember that steatosis is not a specific feature of liver disease, but a histologic finding that can be identified in various other liver biopsies for elevated liver tests. In fact, zone 1 steatosis is a common distribution in chronic hepatitis $\mathrm{C}$. The pathologist should always be alert to other possibilities, particularly when the clinical evaluation is incomplete.

Many patients biopsied to evaluate NAFLD will have a significant degree of steatosis, but will not have evidence of steatohepatitis. There is macrovesicular steatosis that is usually present in a zone 3 or panacinar distribution $\left(\right.$ - Fig. 1). ${ }^{7}$ Steatosis in a zone 1 distribution should suggest the zone 1, borderline pattern. Azonal steatosis is most often seen in biopsies with advanced fibrosis, but the zonal distribution may also be difficult to discern when the steatosis is either very mild or the biopsy is fragmented or inadequate. ${ }^{7}$ Spotty lobular inflammation is often present and can be in the form of small collections of macrophages (microgranulomas) or lymphocytes, similar to the spotty inflammation in chronic 


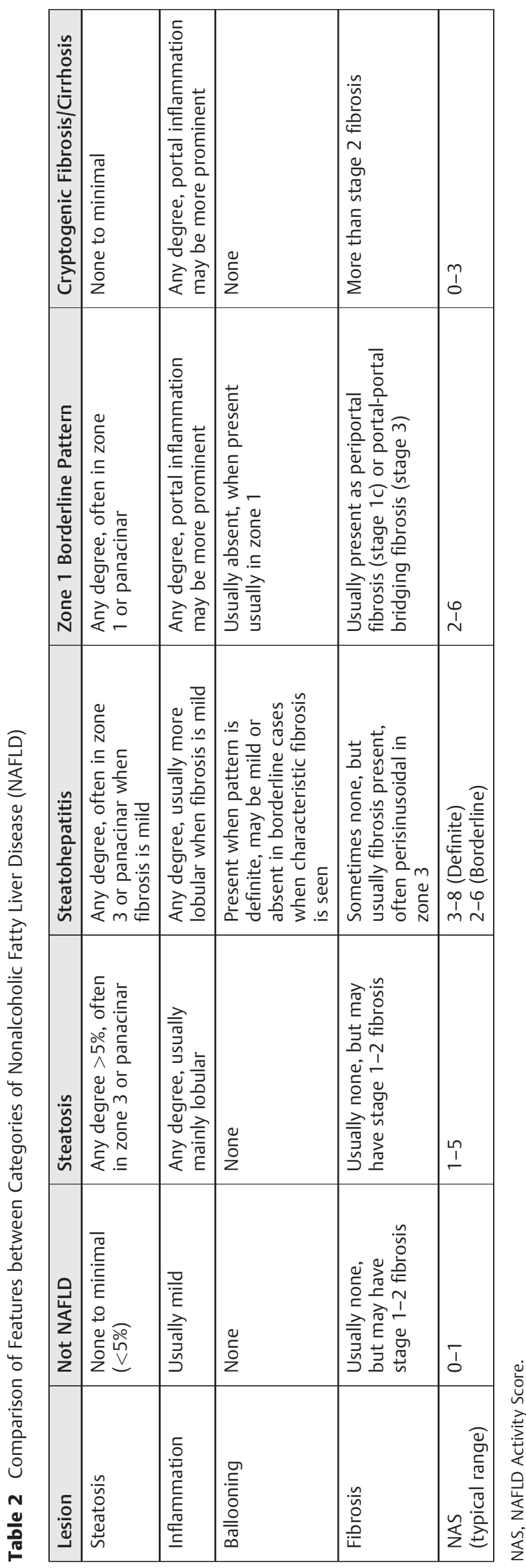

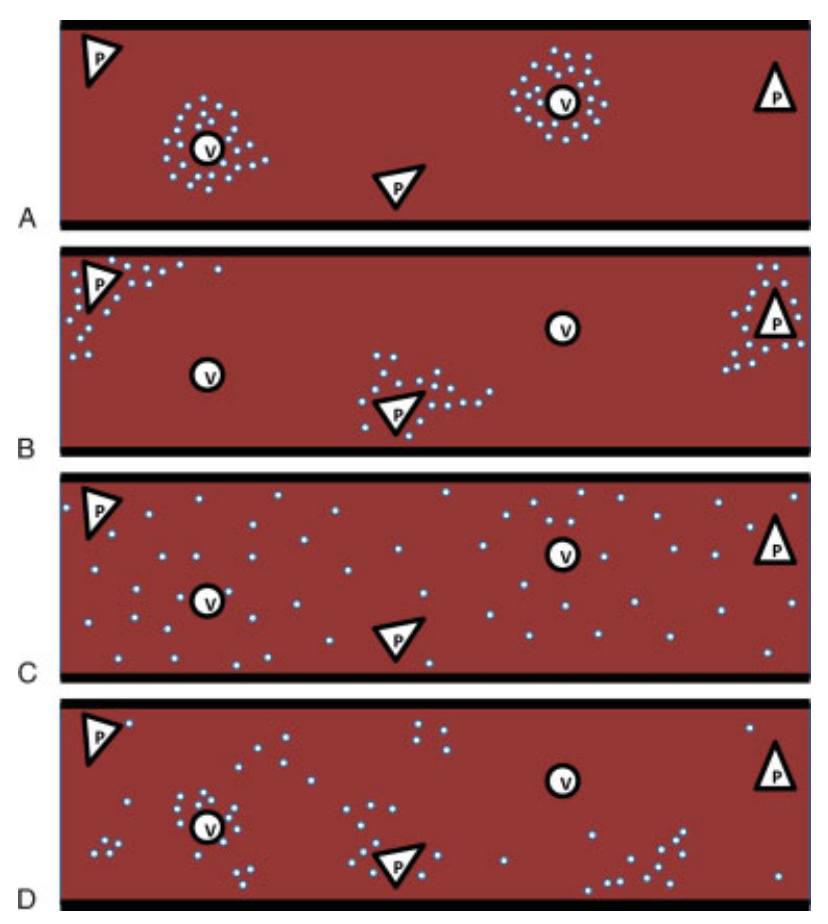

Figure 1 Distribution patterns of steatosis in nonalcoholic fatty liver disease. Shown are the four distribution patterns of steatosis defined in the Non-alcoholic Steatohepatitis Clinical Research Network feature scoring system. The red rectangles represent stylized liver biopsies with triangular portal areas $(\mathrm{P})$ and round central veins $(\mathrm{V})$. The small white circles denote steatotic hepatocytes. The amount of steatosis is the same in each pattern and is relatively mild for demonstration purposes. (A) Zone 3 steatosis. (B) Zone 1 steatosis. (C) Panacinar steatosis. (D) Azonal steatosis.

viral hepatitis. Portal inflammation is usually mild or absent; no ballooning injury suggests steatohepatitis. Fibrosis, if present at all, should be limited to mild periportal or perisinusoidal fibrosis. In the Central Pathology Committee of the NASH CRN, this category has been used for cases in which there is clear zone 3 steatosis with or without periportal fibrosis, but without perisinusoidal fibrosis. In such cases, the criteria for steatohepatitis are incomplete, and the temporal relationship of steatosis and the fibrosis is not known. Because a biopsy with findings in this category shows generally nonspecific findings, a descriptive diagnosis and comment would be appropriate for the pathologist's report.

\section{Steatohepatitis}

Steatohepatitis, as a name for a unique diagnostic pattern that had been observed in nonalcoholic, overweight adult patients, was first proposed in a landmark study by the Mayo Clinic. ${ }^{8}$ However, the alcoholic-like pathology of steatohepatitis was described in overweight and/or diabetic patients long before the study that popularized the term, steatohepatitis (see, e.g., early studies by Zimmerman ${ }^{9,10}$ ). Until recently, it was the only pattern within NAFLD that was recognized to be associated with the development of advanced fibrosis. Unlike the steatosis category described above, steatohepatitis is a specific pattern of liver injury that may be recognized 
even though a second liver disease may be present. The presence of a characteristic ballooning injury is the key to the diagnosis. Ballooning injury is a type of cellular change in which the cells become enlarged and the cytoplasm becomes irregularly clumped with optically clear, nonvesiculated areas. Steatotic vacuoles may be seen in ballooned cells, but they should not fill the cytoplasm. Early in the disease, ballooned cells are seen most frequently in zone 3 near the hepatic veins, but later in the disease or in very severe cases, they may lose this clear zonal distribution. The most classic appearing balloon cells will contain Mallory-Denk bodies (MDBs), which are eosinophilic, ropey cytoplasmic inclusions often found near the nucleus. MDBs are composed of hyperphosphorylated, misfolded cytokeratin 8 and 18 filaments. ${ }^{11}$ Normally, such aggregates would be degraded in the proteosome, but in steatohepatitis this process is blocked. MDBs stain positively using antibodies against ubiquitin and p63, both proteins involved in degradation pathways, as well as with antibodies against cytokeratins 8 and $18 .^{12}$ Using the latter stain, the remaining cytoplasm in the cell will be negative, whereas nonballooned hepatocytes will remain diffusely stained.

Although the term steatohepatitis implies that steatosis and inflammation are important parts of the injury, these components may vary considerably in degree. A biopsy that shows characteristic ballooning injury, but has little steatosis or inflammation, may still be confidently classified as steatohepatitis. Early in the disease, when fibrosis is mild or absent, the steatosis typically shows a zone 3 distribution; the inflammation is predominantly lobular and associated with the steatosis and ballooning. ${ }^{7}$ As the disease progresses, the steatosis may lose this classical zonal distribution and the portal inflammation may become more prominent. The lobular inflammation may become so severe in zone 3 that the pathologist may mistake inflamed perivenular areas for inflamed portal areas and classify the biopsy as "chronic hepatitis." This pitfall can be avoided by looking for the balloon cells, which are usually present in such cases as well as the absence of the interlobular bile duct. As has been recently documented, in severe inflammation, an arteriole may be prominent in zone $3 .^{13}$

The pattern of fibrosis is also distinctive in steatohepatitis. Although not required to make a diagnosis of steatohepatitis, fibrosis is usually present and its presence can be very helpful. Fibrosis begins in zone 3 as delicate strands of collagen that isolate one or more hepatocytes. A good quality Masson trichrome stain (or equivalent) is critical to find the earliest evidence of fibrosis. Later, periportal fibrosis will develop, with strands of collagen entrapping periportal hepatocytes and extending into the surrounding parenchyma. At this stage, a component of ductular reaction may be observed. Rarely, a case with characteristic ballooning injury will show only periportal fibrosis-these cases should still be classified as steatohepatitis. As the disease progresses, bridging fibrosis will form. Unlike viral hepatitis, in which the fibrotic bridges are usually single, thick, collagen septations of collapsed parenchyma and ductular reaction, the bridges that form first in steatohepatitis may be extensions of the perisinusoidal fibrosis that forms networks between adjacent vascular structures. Bridging fibrosis may be centralcentral, with retention of hepatocytes within the complex network of fibrosis; may be central-portal, with eventual loss of hepatocytes, or may be portal-portal in which parenchymal loss and ductular reaction comprise the septal structures. Eventually, the trapped hepatocytes will be lost, and regeneration will create solid nodules of hepatocytes, so that the end stage may resemble cirrhosis resulting from other forms of nonbiliary liver disease. Because the fibrotic progression in steatohepatitis involves both portal areas and hepatic veins (-Fig. 2), fibrosis staging systems used for viral hepatitis are inadequate and fibrosis modeling is nonlinear.

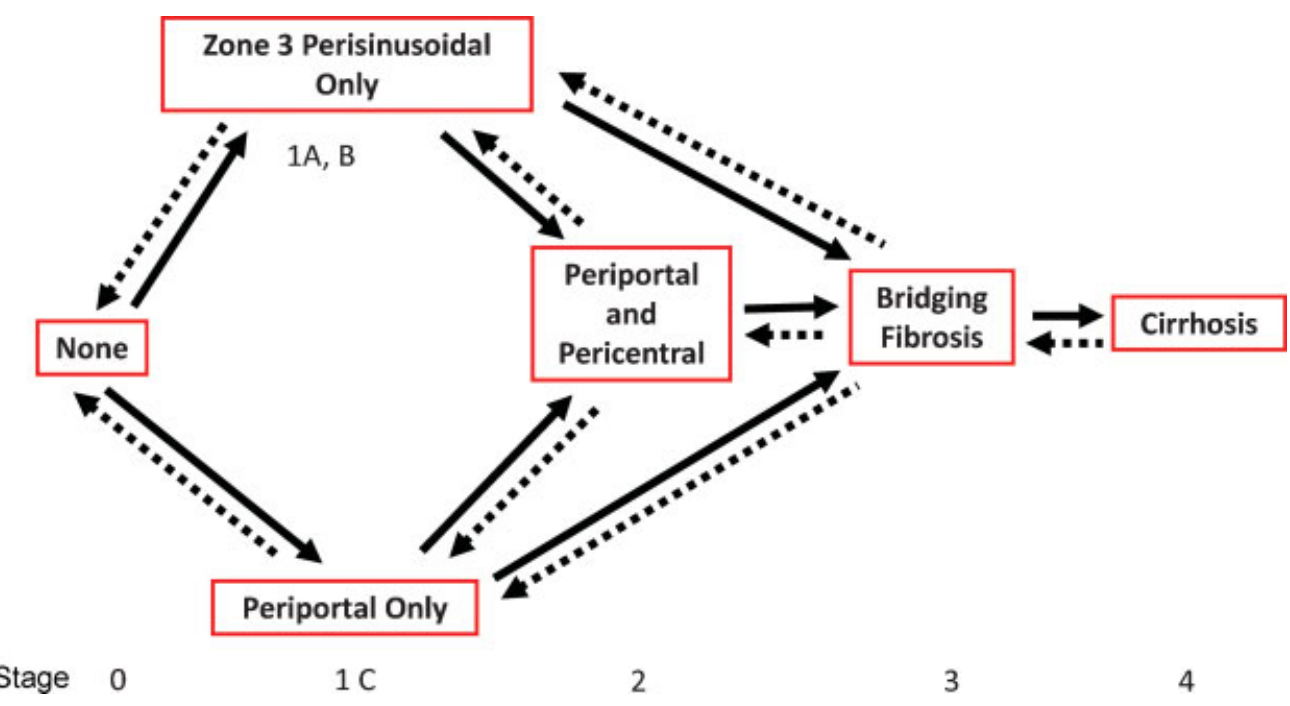

Figure 2 Possible fibrosis progression pathways in steatohepatitis. Fibrosis progression in steatohepatitis is potentially more complicated than in the chronic viral hepatitides. Solid arrows depict possible paths of progression, whereas hatched arrows indicate possible paths of regression. Stages of fibrosis in the Non-alcoholic Steatohepatitis Clinical Research Network system are shown beneath the corresponding texts. 
When the histologic pattern is not distinct enough to make an unequivocal diagnosis of steatohepatitis, but there are still suggestive changes, the term, borderline steatohepatitis has been utilized. ${ }^{14,15}$ In the NASH CRN it has been the practice of the Central Pathology Committee to use this category for cases with characteristic fibrosis, but in which no characteristic ballooning injury is found. There is some variation in practice on this point and many pathologists feel comfortable making the diagnosis of steatohepatitis if characteristic fibrosis is present in the absence of ballooning. In other cases, in which the changes of ballooning and/or fibrosis are mild or equivocal, borderline steatohepatitis may be a better diagnosis than either definite steatohepatitis or steatosis alone. Studies from the NASH CRN have demonstrated that patients with biopsies that show borderline steatohepatitis have clinical characteristics that fall between those with definite steatohepatitis and those classified as not having steatohepatitis. ${ }^{16}$

Other histologic features may be seen in biopsies of patients with NAFLD, including nonzonal small patches of hepatocytes with distinctly microvesicular steatosis, megamitochondria, glycogenated nuclei, large lipogranulomas, and hepatocellular glycogenosis, but these findings have little bearing on the categorization of NAFLD or the diagnosis of steatohepatitis. The cytoplasmic changes of microvesicular steatosis and glycogenosis deserve special mention. Microvesicular steatosis results in a distinct alteration in hepatocytes: under low power magnification, the hepatocytes are pale, but on higher magnification, an almost foamy appearance is noted. Although tiny vesicles may be appreciated, it is a challenge to actually discern individual fat vesicles, and one would not be able to count them. The nucleus is commonly central in the cell. This form of steatosis is quite distinct from the small droplets that can be seen coalescing around the single large vesicle in macrovesicular steatosis, or the occasional droplets within hepatocytes that do not alter the appearance of the cytoplasm otherwise. Glycogenotic hepatocytes may mimic ballooned hepatocytes. The glycogen lakes do not stain well with hematoxylin or eosin and may cause the cytoplasm to have the clumped appearance of true ballooning injury. Apoptotic hepatocytes are not included in the classification of steatohepatitis, but have been shown in several studies and by a variety of methods to correlate with severity of steatohepatitis.

Notably absent in noncirrhotic NAFLD biopsies are the characteristic histologic features associated with alcoholic hepatitis. In alcoholic hepatitis, steatosis is not necessarily present, MDB-containing hepatocytes may not be ballooned and may be surrounded by polymorphonuclear leukocytes (satellitosis); dense perisinusoidal fibrosis and not uncommonly, bilirubinostasis are also present. The prognostic lesion of sclerosing hyaline necrosis of alcoholic hepatitis is not seen in NAFLD. However, in most cases it will not be possible to distinguish alcoholic from nonalcoholic steatohepatitis based on the liver biopsy alone. ${ }^{1,17}$

\section{Zone 1, Borderline Pattern}

In 2005, Schwimmer and colleagues published a series of cases of NAFLD in children. ${ }^{18}$ Although some of the children had steatosis alone and some had steatohepatitis similar to

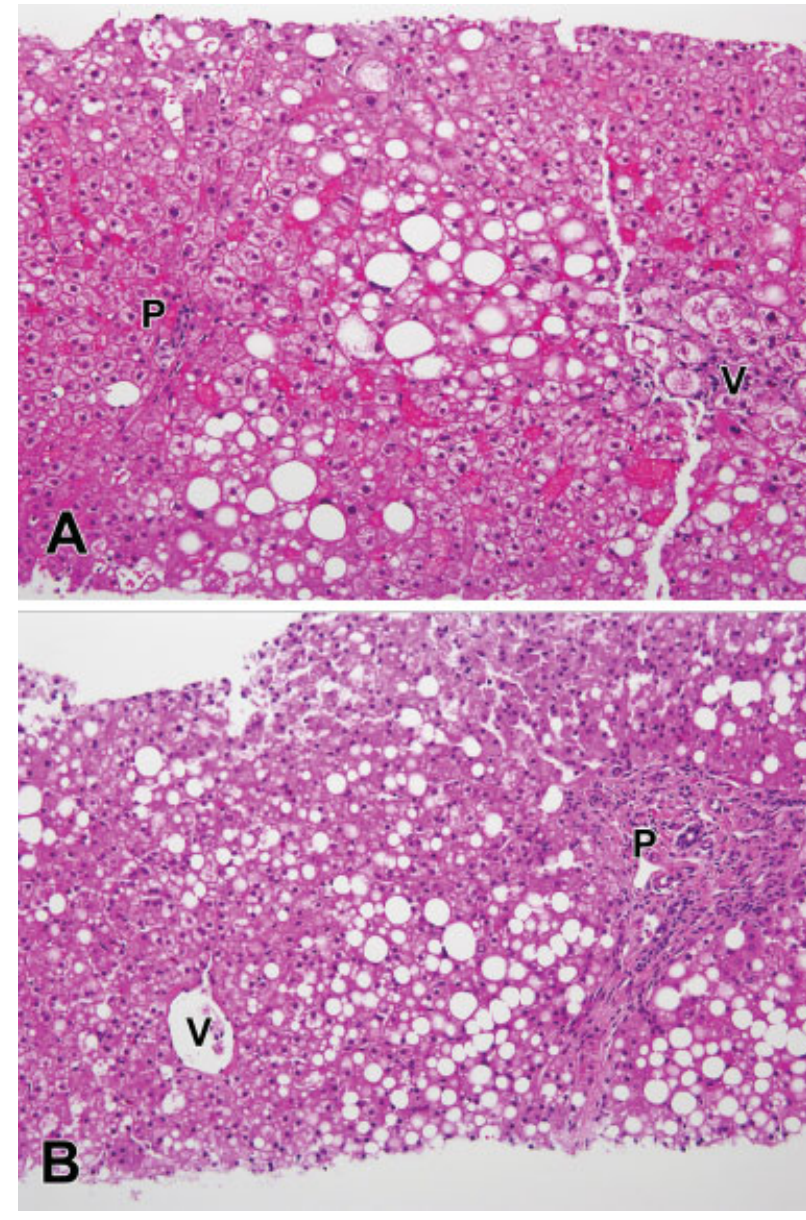

Figure 3 Comparison of steatohepatitis to the zone 1 borderline pattern. In both photomicrographs, portal areas are indicated as "P" and central veins are indicated by "V." (A) Steatohepatitis. There is clear ballooning injury near the central vein, with inflammation and steatosis nearby. The region near the portal area is devoid of these findings. (B) Zone 1 borderline pattern. The portal area is expanded by fibrosis and chronic inflammation. Steatosis completely surrounds the portal area. In contrast, the central vein region shows none of these findings.

that seen in adults, some had a previously unrecognized pattern of fibrotic fatty liver disease in which the injury seemed to involve acinar zone 1 rather than acinar zone 3 . Since that time, these observations have been extended by the NASH CRN and others with an incidence varying from 8 to $22 \%$ in the populations studied. ${ }^{19,20}$ Within the NASH CRN, the pathology committee separated out these cases as "borderline, zone 1, steatohepatitis."19 The borderline term has been used to indicate evidence of a potentially fibrotically progressive NAFLD that lacked the full spectrum of features of typical definite steatohepatitis. This pattern is seen most often in younger, usually pre- or peripubertal children and rarely in older teens and adults. Biopsies show steatosis that is either zone 1 predominant or panacinar; in either case, the steatosis is closely associated with portal areas and tends to diminish around the hepatic veins ( - Fig.3). Ballooning injury is unusual and MDB are essentially never seen in this pattern. Portal inflammation is present, but usually mild, with little or 
no interface hepatitis. Because of the lack of ballooning, the presence of fibrosis is the only feature that sets these cases apart from the nonspecific pattern of steatosis described above. The fibrosis is initially periportal and progresses to portal-portal bridging fibrosis, with distinct sparing of the hepatic veins. The natural history of the zone 1 , borderline pattern is unclear due to the lack of longitudinal studies that include patients with this pattern of injury. It should be noted that none of the existing imaging methods that can identify steatosis or fibrosis in the liver would be able to distinguish this zone 1 , borderline pattern from classical steatohepatitis.

\section{Cryptogenic Fibrosis and Cirrhosis}

Because of the temporally variable nature of steatosis and the tendency of the characteristic lesions of ballooning, MDB and perisinusoidal fibrosis to disappear along with acinar architecture as the disease progresses, there will be some cases of advanced liver disease that lack sufficient changes to be classified into one of the four groups outlined above. Histologically, there is advanced fibrosis present, usually cirrhosis but sometimes only bridging fibrosis, minimal steatosis and no features that would suggest steatohepatitis, such as ballooning injury or MDBs. When this occurs because of therapy and a preceding biopsy shows steatohepatitis, a descriptive diagnosis that includes the phrase "consistent with resolved steatohepatitis" could be used. When either cryptogenic fibrosis or cirrhosis is present at baseline, a diligent search should be made to exclude other causes of advanced liver disease before ascribing the case to previous fatty liver disease that has now disappeared, as it is well-recognized that other forms of chronic liver disease, such as alcoholic cirrhosis and autoimmune hepatitis, may also "burn out."

\section{Evaluation of Liver Biopsies in NAFLD}

\section{Adequacy}

Prior to evaluation of liver biopsies in any form of medical liver disease, the pathologist must make a determination of adequacy of sampling. This continues to be most relevant in NAFLD due to the fact that clinical testing can only result in exclusion of other liver disease and a strong likelihood of NAFLD, but not a definitive diagnosis. For adequacy, therefore, the biopsy must penetrate sufficiently below the capsule and into hepatic parenchyma to avoid a purely subcapsular sample. Length of the sample can be considered a surrogate parameter of adequacy; $1.5 \mathrm{~cm}$ is generally considered appropriate. ${ }^{21}$ The width of the biopsy is also important, as narrow bore biopsies may not show complete acini and may transect portal tracts, leaving the pathologist without a firm assessment of architectural integrity.

Just as with other forms of chronic liver disease, the process of NAFLD may not necessarily affect the liver parenchyma uniformly. ${ }^{22}$ However, a variety of techniques can be utilized to minimize sampling "error." These are particularly important in clinical studies that include serial biopsies. The biopsies should be obtained from subjects in a similar fashion; otherwise, a certain degree of variation may be due to the difference in technique. These include the use of large bore needles (16 gauge or wider) and a needle biopsy length of at least $1.5 \mathrm{~cm}$ to $2.0 \mathrm{~cm} \cdot{ }^{23,24}$ Recognition of the differences of anatomy between the lobes highlights the potential for greater fibrosis in left lobe biopsies, due to larger portal structures near the capsule and higher capsule : parenchyma ratio compared with a right lobe biopsy. Biopsies obtained during ratio surgical procedure will result in clusters of polymorphonuclear leukocytes (known to pathologists as surgical hepatitis) if care is not taken to perform the biopsy as quickly as possible after induction of anesthesia. ${ }^{25}$ This may result in inability to appropriately judge inflammation. Failure to place the biopsy immediately in fixative may result in autolysis, which will lower estimates of inflammation due to lysis of lymphocytes. Finally, the significance of pathologist expertise has been documented in this disease, ${ }^{26}$ just as in other forms of chronic liver disease. ${ }^{27}$

\section{Grading and Staging}

The concept of grading and staging the histologic features in NAFLD are theoretically similar to those for chronic hepatitis: grade is a global measure of hepatocellular and necroinflammatory injury and reflects features that are potentially reversible. Stage is an assessment of fibrosis location (i.e., scar) and architectural remodeling; thus, it is potentially irreversible. A subtle, but important distinction between grade and stage, is the former describes amount, whereas the latter does not. Stage, in all scoring systems to date, describes only parenchymal location of collagen and matrix deposition, and vascular/architectural alterations, but not absolute quantity. ${ }^{28,29}$ Morphometric measurements of fibrosis in liver disease require specialized techniques, which yield important, but different information than staging. Descriptors such as mild, moderate, and severe can be utilized for grade, whereas these should not be applied to stage because of the inclusion of fibrosis location (zone 3 perisinusoidal, for example), and architectural alteration(s) when present (i.e., cirrhosis).

In 1999, a classification of NAFLD was proposed ${ }^{30}$ that combined the necroinflammatory lesions and fibrosis. That same year, a method of grading and staging NASH was proposed. ${ }^{31}$ The two studies served separate purposes; the latter was modeled upon the contemporaneous paradigms in chronic hepatitis that separately assessed features of ongoing injury (i.e., activity) and scarring (i.e., fibrosis; reviewed in ${ }^{32}$ ). The 1999 proposal specifically focused on lesions that differed from those of portal-based chronic hepatitis scoring systems. The NASH CRN further developed a semiquantitative scoring system for evaluation of serial biopsies by routine hematoxylin and eosin and Masson trichrome stains from patients in clinical trials. ${ }^{5}$ In this system, three features were identified in a blinded, validated study that correlated with the separately derived diagnosis of steatohepatitis/steatosis amount, lobular inflammation amount, and ballooning injury severity. The lesions are individually scored and the unweighted sum is the NAFLD Activity Score (NAS). The fibrosis score, as discussed and diagrammed in -Fig. 2, reflects the unique patterns of fibrosis that may occur in the variety of 
Table 3 Grading and Staging

\begin{tabular}{|c|c|c|c|c|c|}
\hline \multirow[b]{2}{*}{ Components to score and add } & \multicolumn{5}{|c|}{ Grade } \\
\hline & Grade 0 & Grade 1 & Grade 2 & Grade 3 & Sum \\
\hline Steatosis & $<5 \%$ (of hepatocytes) & $5-33 \%$ & $34-66 \%$ & $>67 \%$ & $0-3$ \\
\hline Lobular inflammation & $0 / 20 x$ & $<2$ foci $/ 20 x$ & $2-4$ foci/20x & $>4$ foci $/ 20 x$ & $0-3$ \\
\hline Ballooning & 0 & Few; inapparent & Easily noted; many & $\mathrm{N} / \mathrm{A}$ & $0-2$ \\
\hline NAFLD activity score (NAS) & & & & & $0-8$ \\
\hline Stage & \multicolumn{2}{|l|}{ Fibrosis location } & \multicolumn{3}{|c|}{ Architectural alterations } \\
\hline $1 \mathrm{~A}$ & \multicolumn{2}{|c|}{ Zone 3, perisinusoidal, delicate } & \multicolumn{3}{|l|}{ No } \\
\hline 1B & \multicolumn{2}{|c|}{ Zone 3, perisinusoidal, dense } & \multicolumn{3}{|l|}{ No } \\
\hline $1 C$ & \multicolumn{2}{|l|}{ Portal, periportal only } & \multicolumn{3}{|c|}{ May see ductular reaction } \\
\hline 2 & \multicolumn{2}{|c|}{ Zone 3 perisinusoidal + portal/periportal } & \multicolumn{3}{|c|}{$\begin{array}{l}\text { May see ductular reaction; may have foci } \\
\text { concerning for bridging }\end{array}$} \\
\hline 3 & \multicolumn{2}{|c|}{ Bridging fibrosis: C-C; C-P; P-P } & \multicolumn{3}{|l|}{ Yes, see text } \\
\hline 4 & \multicolumn{2}{|l|}{ Cirrhosis } & \multicolumn{3}{|l|}{ Yes, see text } \\
\hline
\end{tabular}

NAFLD, Nonalcoholic fatty liver disease; NAS, NAFLD Activity Score.

conditions of NAFLD. - Table 3 summarizes the NAS and fibrosis scores.

\section{NAS vs NASH: Or...NAS: Can It Be Considered an Appropriate Replacement for the Diagnosis of NASH?} Prior to the discovery of the hepatitis $C$ virus and the widespread epidemic of obesity and associated dysregulated lipids, liver test elevations could not always be explained by serologic assays, however, there was a recognition that "chronic hepatitis" existed and had prototypic characteristic histopathologic features regardless of specific etiology. For example, regardless of pathogenesis, "chronic hepatitis" is characterized by portal chronic inflammation that breeches the limiting plate (i.e., interface activity). One of the first methods of semiquantitative evaluation was created to assess treatment efficacy; this system was popularized as the Knodell Histologic Activity Index. ${ }^{33}$ Scores for activity could range between 0 to 14 , and those for fibrosis, 0 to 4 . Interestingly, no single HAI score was chosen as a diagnostic score of chronic hepatitis; the diagnosis remains up to the pathologist after clinical inclusion/exclusion by serologic testing.

When the NAS (0-8) was proposed, the study was based on 32 adult biopsies read blinded twice by nine pathologists, and clearly documented that biopsies with higher scores $(\geq 5)$ tended to be diagnosed as steatohepatitis, whereas biopsies with lower scores $(\leq 3)$ were generally not considered to be diagnostic of steatohepatitis. ${ }^{5}$ However, there were several biopsies in the NAS 3 to 5 range that overlapped all diagnostic criteria. The relationship between the score and the diagnosis is a consequence of how the score was created because the score incorporated major histologic features (aside from fibrosis) that pathologists use to make the diagnosis of steatohepatitis. The NASH CRN has published a more recent study of 934 noncirrhotic adult biopsies scored by the Central Pathology Committee and contemporaneous demographic and laboratory features. The findings support the concepts that the NAS and the diagnosis are interrelated, but also do in fact have distinct clinicopathologic meanings; thus, they should not be considered interchangeable. ${ }^{34}$ Among cases with NAS $\geq 5,7 \%$ were diagnosed as "not steatohepatitis" and $38 \%$ as "borderline." In the definite steatohepatitis category, only $75 \%$ were NAS $\geq 5$, thus the remaining $25 \%$ had scores $\leq 4$. The sensitivity and specificity of NAS $\geq 5$ for definite steatohepatitis were 0.75 and 0.83 , respectively. By multivariable regression, it was shown that although both NAS and the diagnosis correlated with elevations of aspartate aminotransferase and alanine aminotransferase, only the diagnosis of steatohepatitis did so with metabolic syndrome, diabetes, and insulin resistance as measured by either homeostasis model assessment-insulin resistance (HOMA-IR) or the Quantitative Insulin Sensitivity Check Index (QUICKI). These findings have important implications for studies that base entry criteria solely on the NAS, rather than on the pathologist's separately derived diagnosis. Such studies risk exclusion of cases with mild but definitive findings of steatohepatitis, and inclusion of cases with marked steatosis and inflammation but lack ballooning.

\section{Other Lesions of Interest in NAFLD/NASH}

\section{Apoptosis}

Apoptotic hepatocytes are readily noted in liver biopsies as rounded, eosinophilic cytoplasmic fragments, which appear to be free within the sinusoids or surrounded by Kupffer or other inflammatory cells. In some instances, the apoptotic hepatocytes may contain pyknotic nuclear material; however, most frequently they do not. Regardless of the method of ascertainment - the terminal deoxynucleotidyltransferase dUTP nick end labeling (TUNEL) assay, serum keratin 18 fragments, or simply recording apoptotic hepatocytes by light microscopy the severity of apoptosis has been shown to correlate with other markers of severity in steatohepatitis. ${ }^{35-38}$ 


\section{Inflammation}

Inflammatory infiltrates can be noted in the hepatic acini/ lobules or the portal tracts. Specifically, the infiltrates are predominantly of the chronic inflammatory cell phenotypes, such as lymphocytes, monocytes, occasional eosinophils, and macrophages/Kupffer cells. Rare polymorphs are noted; however, a lesion common to many forms of alcoholic hepatitis, referred to as satellitosis because of polymorphs encircling MDB-containing hepatocytes, is rare in NASH. Likewise, the portal-based lesion of ductular reaction accompanied by fibrosis and periductular polymorphs, pericholangitis, which frequently occurs in alcoholic hepatitis, ${ }^{1}$ is a feature that would raise a concern of pancreatitis or even alcoholic injury in NASH. On the other hand, lipogranulomas of varying sizes and distribution are common to both NAFLD and alcoholic liver disease. Lipogranulomas not only consist of varying amounts of vacuolated fat droplets, but also of admixtures of mononuclear or eosinophilic white cells and varying degrees of collagen. Lipogranulomas near a terminal hepatic venule may initially raise a consideration of perisinusoidal fibrosis without careful evaluation. ${ }^{6,39}$

Inflammation in portal tracts may or may not appear to correlate with that in the lobules. In fact, chronic inflammation that is disproportionate to the above-described lesions of activity deserves evaluation for a concurrent form of chronic liver disease, ${ }^{40-42}$ as the recognized prevalence of NAFLD ${ }^{43}$ does not preclude that of the other liver diseases, such as hepatitis $\mathrm{C}$ virus (HCV), autoimmune hepatitis, hemochromatosis, and even ALD, to name a few. There are various proposals in the pathology literature as to how to approach a liver biopsy with a documented form of chronic liver disease and NAFLD. Documentation of amount and location of steatosis may or may not serve as a clue to the "origin" of the steatosis, as steatosis in HCV gt non3 is commonly periportal, in contrast to perivenular in NAFLD. One group has suggested that ballooning is sufficient to differentiate NASH from $\mathrm{HCV}^{44}$ Another group has suggested stricter criteria, i.e., the presence of zone 3 pericellular fibrosis as the lesion of distinction of steatohepatitis from portal-based fibrosis of other forms of chronic liver disease. ${ }^{40}$ The former will result in higher incidence of steatohepatitis than the latter, as hepatocellular ballooning is a lesion known to occur in viral hepatitis. ${ }^{45}$

Residual portal chronic inflammation was noted to be greater in relative proportion to lobular inflammation in liver biopsies in otherwise successfully treated subjects in a small medical treatment trial. ${ }^{46}$ This finding, although not directly noted, can be found in other reports of surgical ${ }^{47}$ and medical treatment trials in adults. ${ }^{48}$ Recently, the large database of biopsies and matching demographic material from the NASH CRN was studied for the significance of greater than mild portal chronic inflammation in baseline biopsies of 728 adults and 205 children. In both groups, the lesion was associated with clinical and histologic features of severity and advanced disease. $^{49}$

\section{Vascular Alterations}

Recent work has highlighted the microscopic observation of the intraacinar branch of the hepatic artery in the perivenular

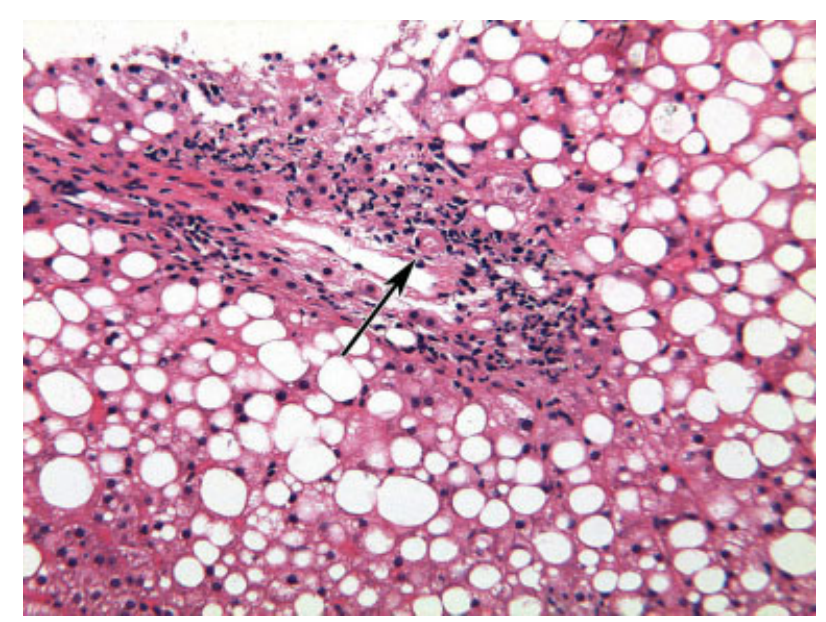

Figure 4 Perivenular fibrosis with a neo-artery. In this case of steatohepatitis, there is a perivenular cuff of inflammation and fibrosis. A small arteriole is present (arrow). No bile duct is seen. This appearance of the central vein may cause an observer to mistakenly conclude that this is a portal area, leading to a diagnosis of chronic hepatitis rather than steatohepatitis.

region in active steatohepatitis (-Fig. 4)..$^{13}$ The finding correlates with severity of disease. The presence of this vessel alongside the outflow vein may lead to confusion for a portal tract, particularly when perivenular inflammation is marked. Thus, careful study to document the presence or absence of an accompanying interlobular bile duct becomes crucial.

\section{Implications of Histologic Evaluation for Designing Treatment Trials}

This topic was the focus of a recent AASLD Endpoints Workshop that was subsequently published. ${ }^{15}$ The definitions as detailed above have been proposed in the article for steatosis and steatohepatitis (definite and borderline). The use of the NASH CRN scoring system has been discussed as a validated method of objective semiquantitative assessment of histologic features that can be compared before and after treatment intervention. The NAS itself has applied in a long-term outcome study of 52 Asian subjects with repeated biopsies at 36 months. The authors found moderate correlation of change in activity score and serum keratin 18 levels, and independent correlation with increase in body mass index in subjects who showed progression over a 3-year period. ${ }^{50}$ Weight reduction was the most important variable in improvement or lack of worsening in both activity and fibrosis.

\section{NAFLD in Lipodystrophy}

Lipodystrophy encompasses a heterogeneous group of diseases with abnormal subcutaneous adipose tissue distribution. The changes in subcutaneous fat may be localized, partial (e.g., affecting major portions of the body), or general. Partial and general forms of lipodystrophy may be the result of congenital genetic mutations or may be acquired, often as a medication side effect. One of the consequences of the loss of adipose tissue is peripheral insulin resistance, as well as decreased serum levels of adiponectin and leptin. Fat may be stored in other organs, including the liver, skeletal muscle, 
and pancreas. ${ }^{51,52}$ Patients with lipodystrophy often have type 2 diabetes mellitus and hypertriglyceridemia. Although hepatic steatosis and steatohepatitis have been recognized in patients with lipodystrophy, most of the data are in the form of case reports and small series. In recent a series of 10 lipodystrophic patients undergoing leptin therapy, liver biopsies were performed before and during therapy to assess whether the systemic metabolic improvements were having the expected effect on the liver. ${ }^{53}$ Of the 10 patients, eight patients had steatohepatitis at baseline, one had only mild steatosis, and one did not have NAFLD at all. Fibrosis was common, with some degree of fibrosis in eight patients, whereas three had bridging fibrosis and one had cirrhosis. The pattern of injury was that of the classical steatohepatitis described above, although only three had MDBs. Treatment with leptin was associated with resolution of histologic and biochemical features of liver disease. Other investigators have also reported steatosis and steatohepatitis on biopsy in patients with lipodystrophy. ${ }^{54,55}$ Ultrastructural studies have demonstrated alterations in mitochondria including abnormal cristae, matrix rarefication, and crystalloids, as well as increased numbers of peroxisomes. ${ }^{56,57}$ Certain antiretroviral therapies used to treat human immunodeficiency virus (HIV) infection have been associated with acquired forms of lipodystrophy and also with NAFLD, and NAFLD is a common finding on liver biopsy in this population. ${ }^{58}$ Because NAFLD and NASH are common liver diseases and HIV-infected patients may also have other predisposing factors, a causal association between individual drugs and liver disease is difficult to establish.

\section{NAFLD in Bariatric Surgery Patients}

Patients undergoing bariatric surgery for weight loss are at risk for NAFLD and often have other comorbidities related to severe obesity, including diabetes mellitus, hypertension, sleep apnea, and coronary artery disease. A high percentage will have metabolic syndrome. Because the left lobe of the liver is accessible to the surgeon during the procedure, biopsy surveys have provided a window into the liver disease of these patients. A meta-analysis of such surveys that included 1620 patients from 12 studies noted a high prevalence of steatosis and steatohepatitis ( $91 \%$ and $37 \%$, respectively). ${ }^{3}$ Although the proportion of patients with steatosis was high across all of the studies (85 to 99\%), the incidence of steatohepatitis varied from 24 to $98 \%$. The incidence of individual findings beyond steatosis varied considerably, with inflammation seen in 24 to $98 \%$ and fibrosis in 13 to $97 \%$ across studies. These differences could represent differences in operational histologic definitions, but may also represent differences in populations undergoing bariatric surgery at the various institutions. Despite the high prevalence of liver disease, biopsy of the liver during the procedure is not standard practice among surgeons. Visible inspection of the liver by the surgeon is not sufficient to exclude liver disease. In a study of 100 patients in which the external appearance of the liver was systematically recorded by the surgeon, there was no relationship between the gross appearance and the presence or severity of liver disease on biopsy. ${ }^{59}$
Several studies have reported an increased prevalence of periportal fibrosis (NASH CRN stage 1C) in morbidly obese patients. Abrams and coworkers first brought attention to this finding. In their cohort of 195 bariatric surgery patients, a third had stage $1 \mathrm{C}$ fibrosis, most with some degree of steatosis and inflammation. ${ }^{60}$ The proportion of early stage fibrosis that is portal as opposed to perisinusoidal is $\sim 50 \%,{ }^{61,62}$ which is a larger proportion than in nonbariatric populations undergoing biopsy to evaluate NAFLD. ${ }^{14}$ The clinical significance of this finding and how it relates to the other features of NAFLD is unclear.

\section{Summary}

When liver biopsies are performed as part of clinical studies of NAFLD, attention should be paid to the overall disease classification according to the pattern of injury as well as to the grade and stage of the disease. Individual lesions can be evaluated for severity and distribution within the acinar architecture of the liver. There may be differences in the histologic features of various populations such as bariatric surgery patients or pediatric patients as compared with adult populations derived from hepatology clinics or diabetes clinics. It is important to note that the process of grading and staging is related to, yet separate from the process of assigning a diagnostic pattern. Both are important in the pathologic evaluation of NAFLD liver biopsies.

\section{Acknowledgment}

This work was supported in part by the Intramural Program of the $\mathrm{NIH}$, National Cancer Institute.

\section{Abbreviations}

$\begin{array}{ll}\text { AASLD } & \begin{array}{l}\text { American Association for the Study of Liver } \\ \text { Diseaseflow } \\ \text { hepatitis C virus } \\ \text { HCV }\end{array} \\ \text { hIV } & \begin{array}{l}\text { human immunodeficiency virus } \\ \text { homeostatic model assessment-insulin } \\ \text { resistance }\end{array} \\ \text { MDB } & \begin{array}{l}\text { Mallory-Denk body } \\ \text { nonalcoholic fatty liver disease }\end{array} \\ \text { NAFLD } & \begin{array}{l}\text { nonalcoholic steatohepatitis } \\ \text { NASH }\end{array} \\ \text { NASH CRN } & \begin{array}{l}\text { Non-alcoholic Steatohepatitis Clinical Research } \\ \text { Network }\end{array}\end{array}$

\section{References}

1 Itoh S, Yougel T, Kawagoe K. Comparison between nonalcoholic steatohepatitis and alcoholic hepatitis. Am J Gastroenterol 1987;82(7):650-654

2 Anty R, Iannelli A, Patouraux S, et al. A new composite model including metabolic syndrome, alanine aminotransferase and cytokeratin-18 for the diagnosis of non-alcoholic steatohepatitis in morbidly obese patients. Aliment Pharmacol Ther 2010;32; (11-12):1315-1322 
3 Machado M, Marques-Vidal P, Cortez-Pinto H. Hepatic histology in obese patients undergoing bariatric surgery. J Hepatol 2006;45 (4):600-606

4 Xanthakos S, Miles L, Bucuvalas J, Daniels S, Garcia V, Inge T. Histologic spectrum of nonalcoholic fatty liver disease in morbidly obese adolescents. Clin Gastroenterol Hepatol 2006;4(2):226232

5 Kleiner DE, Brunt EM, Van Natta M, et al; Nonalcoholic Steatohepatitis Clinical Research Network. Design and validation of a histological scoring system for nonalcoholic fatty liver disease. Hepatology 2005;41(6):1313-1321

6 Burt AD, Mutton A, Day CP. Diagnosis and interpretation of steatosis and steatohepatitis. Semin Diagn Pathol 1998;15(4): 246-258

7 Chalasani N, Wilson L, Kleiner DE, Cummings OW, Brunt EM, Unalp A; NASH Clinical Research Network. Relationship of steatosis grade and zonal location to histological features of steatohepatitis in adult patients with non-alcoholic fatty liver disease. J Hepatol 2008;48(5):829-834

8 Ludwig J, Viggiano TR, McGill DB, Oh BJ. Nonalcoholic steatohepatitis: Mayo Clinic experiences with a hitherto unnamed disease. Mayo Clin Proc 1980;55(7):434-438

9 Zimmerman HJ, MacMurray FG, Rappaport H, Alpert LK. Studies on the liver in diabetes mellitus. II. The significance of fatty metamorphosis and its correlation with insulin sensitivity. J Lab Clin Med 1950;36(6):922-928

10 Zimmerman HJ, MacMurray FG, Rapparport H, Alpert LK. Studies of the liver in diabetes mellitus. I. Structural and functional abnormalities. J Lab Clin Med 1950;36(6):912-921

11 Omary MB, Ku NO, Strnad P, Hanada S. Toward unraveling the complexity of simple epithelial keratins in human disease. J Clin Invest 2009;119(7):1794-1805

12 Lackner C, Gogg-Kamerer M, Zatloukal K, Stumptner C, Brunt EM, Denk $\mathrm{H}$. Ballooned hepatocytes in steatohepatitis: the value of keratin immunohistochemistry for diagnosis. J Hepatol 2008;48 (5):821-828

13 Gill RM, Belt P, Wilson L, Bass NM, Ferrell LD. Centrizonal arteries and microvessels in nonalcoholic steatohepatitis. Am J Surg Pathol 2011;35(9):1400-1404

14 Neuschwander-Tetri BA, Clark JM, Bass NM, et al; NASH Clinical Research Network. Clinical, laboratory and histological associations in adults with nonalcoholic fatty liver disease. Hepatology 2010;52(3):913-924

15 Sanyal AJ, Brunt EM, Kleiner DE, et al. Endpoints and clinical trial design for nonalcoholic steatohepatitis. Hepatology 2011;54 (1):344-353

16 Patton HM, Lavine JE, Van Natta ML, Schwimmer JB, Kleiner D, Molleston J; Nonalcoholic Steatohepatitis Clinical Research Network. Clinical correlates of histopathology in pediatric nonalcoholic steatohepatitis. Gastroenterology 2008;135(6):19611971, e2

17 Diehl AM, Goodman Z, Ishak KG. Alcohollike liver disease in nonalcoholics. A clinical and histologic comparison with alcoholinduced liver injury. Gastroenterology 1988;95(4):1056-1062

18 Schwimmer JB, Behling C, Newbury R, et al. Histopathology of pediatric nonalcoholic fatty liver disease. Hepatology 2005;42 (3):641-649

19 Patton HM, Yates K, Unalp-Arida A, et al. Association between metabolic syndrome and liver histology among children with nonalcoholic fatty liver disease. Am J Gastroenterol 2010;105 (9):2093-2102

20 Carter-Kent C, Yerian LM, Brunt EM, et al. Nonalcoholic steatohepatitis in children: a multicenter clinicopathological study. Hepatology 2009;50(4):1113-1120

21 Rockey DC, Caldwell SH, Goodman ZD, Nelson RC, Smith AD; American Association for the Study of Liver Diseases. Liver biopsy. Hepatology 2009;49(3):1017-1044
22 Ratziu V, Charlotte F, Heurtier A, et al; LIDO Study Group. Sampling variability of liver biopsy in nonalcoholic fatty liver disease. Gastroenterology 2005;128(7):1898-1906

23 Chalasani NP, Sanyal AJ, Kowdley KV, et al; NASH CRN Research Group. Pioglitazone versus vitamin E versus placebo for the treatment of non-diabetic patients with non-alcoholic steatohepatitis: PIVENS trial design. Contemp Clin Trials 2009; 30(1):88-96

24 Larson SP, Bowers SP, Palekar NA, Ward JA, Pulcini JP, Harrison SA. Histopathologic variability between the right and left lobes of the liver in morbidly obese patients undergoing Roux-en-Y bypass. Clin Gastroenterol Hepatol 2007;5(11):13291332

25 Mattar SGMDF, Velcu LMMD, Rabinovitz MMD, et al. Surgicallyinduced weight loss significantly improves nonalcoholic fatty liver disease and the metabolic syndrome. Ann Surg 2005;242(4):610617, discussion 618-620

26 Vuppalanchi R, Unalp A, Van Natta ML, et al. Effects of liver biopsy sample length and number of readings on histologic yield for nonalcoholic fatty liver disease. Clin Gastroenterol Hepatol 2009; 7:481-486

27 Rousselet M-C, Michalak S, Dupré F, et al; Hepatitis Network 49. Sources of variability in histological scoring of chronic viral hepatitis. Hepatology 2005;41(2):257-264

28 Goodman ZD, Stoddard AM, Bonkovsky HL, et al; HALT-C Trial Group. Fibrosis progression in chronic hepatitis C: morphometric image analysis in the HALT-C trial. Hepatology 2009;50(6): $1738-1749$

29 Fontana RJ, Goodman ZD, Dienstag JL, et al; HALT-C Trial Group. Relationship of serum fibrosis markers with liver fibrosis stage and collagen content in patients with advanced chronic hepatitis C. Hepatology 2008;47(3):789-798

30 Matteoni CA, Younossi ZM, Gramlich T, Boparai N, Liu YC, McCullough AJ. Nonalcoholic fatty liver disease: a spectrum of clinical and pathological severity. Gastroenterology 1999;116 (6):1413-1419

31 Brunt EM, Janney CG, Di Bisceglie AM, Neuschwander-Tetri BA, Bacon BR. Nonalcoholic steatohepatitis: a proposal for grading and staging the histological lesions. Am J Gastroenterol 1999;94 (9):2467-2474

32 Brunt EM. Grading and staging the histopathological lesions of chronic hepatitis: the Knodell histology activity index and beyond. Hepatology 2000;31(1):241-246

33 Knodell RG, Ishak KG, Black WC, et al. Formulation and application of a numerical scoring system for assessing histological activity in asymptomatic chronic active hepatitis. Hepatology 1981;1(5): 431-435

34 Brunt EM, Kleiner DE, Wilson L, et al. The NAS and the histopathologic diagnosis of NASH: distinct clinicopathologic meanings. Hepatology 2011;53:810-820

35 Feldstein AE, Wieckowska A, Lopez AR, Liu YC, Zein NN McCullough AJ. Cytokeratin-18 fragment levels as noninvasive biomarkers for nonalcoholic steatohepatitis: a multicenter validation study. Hepatology 2009;50(4):1072-1078

36 Ramalho RM, Cortez-Pinto H, Castro RE, et al. Apoptosis and Bcl-2 expression in the livers of patients with steatohepatitis. Eur J Gastroenterol Hepatol 2006;18(1):21-29

37 Ribeiro PS, Cortez-Pinto H, Solá S, et al. Hepatocyte apoptosis, expression of death receptors, and activation of NF-kappaB in the liver of nonalcoholic and alcoholic steatohepatitis patients. Am J Gastroenterol 2004;99(9):1708-1717

38 Wieckowska A, Zein NN, Yerian LM, Lopez AR, McCullough AJ, Feldstein AE. In vivo assessment of liver cell apoptosis as a novel biomarker of disease severity in nonalcoholic fatty liver disease. Hepatology 2006;44(1):27-33

39 Yip WW, Burt AD. Alcoholic liver disease. Semin Diagn Pathol 2006;23(3-4):149-160 
40 Brunt EM, Ramrakhiani S, Cordes BG, et al. Concurrence of histologic features of steatohepatitis with other forms of chronic liver disease. Mod Pathol 2003;16(1):49-56

41 Clouston AD, Powell EE. Interaction of non-alcoholic fatty liver disease with other liver diseases. Best Pract Res Clin Gastroenterol 2002;16(5):767-781

42 Sanyal AJ, Contos MJ, Sterling RK, et al. Nonalcoholic fatty liver disease in patients with hepatitis $C$ is associated with features of the metabolic syndrome. Am J Gastroenterol 2003;98(9):2064-2071

43 Younossi ZM, Stepanova M, Afendy M, et al. Changes in the prevalence of the most common causes of chronic liver diseases in the United States from 1988 to 2008. Clin Gastroenterol Hepatol 2011;9(6):524-530, e1, quiz e60

44 Bedossa P, Moucari R, Chelbi E, et al. Evidence for a role of nonalcoholic steatohepatitis in hepatitis C: a prospective study. Hepatology 2007;46(2):380-387

45 Ishak KG. Pathologic features of chronic hepatitis. A review and update. Am J Clin Pathol 2000;113(1):40-55

46 Neuschwander-Tetri BA, Brunt EM, Wehmeier KR, Oliver D, Bacon BR. Improved nonalcoholic steatohepatitis after 48 weeks of treatment with the PPAR-gamma ligand rosiglitazone. Hepatology 2003;38(4):1008-1017

47 Marceau P, Biron S, Hould FS, et al. Liver pathology and the metabolic syndrome $\mathrm{X}$ in severe obesity. J Clin Endocrinol Metab 1999;84(5):1513-1517

48 Promrat K, Lutchman G, Uwaifo GI, et al. A pilot study of pioglitazone treatment for nonalcoholic steatohepatitis. Hepatology 2004;39(1):188-196

49 Brunt EM, Kleiner DE, Wilson LA, et al; NASH Clinical Research Network. A list of members of the Nonalcoholic Steatohepatitis Clinical Research Network can be found in the Appendix. Portal chronic inflammation in nonalcoholic fatty liver disease (NAFLD): a histologic marker of advanced NAFLD-clinicopathologic correlations from the nonalcoholic steatohepatitis clinical research network. Hepatology 2009;49(3):809-820

50 Wong VW-S, Wong GL-H, Choi PC-L, et al. Disease progression of non-alcoholic fatty liver disease: a prospective study with paired liver biopsies at 3 years. Gut 2010;59(7):969-974
51 Monajemi H, Stroes E, Hegele RA, Fliers E. Inherited lipodystrophies and the metabolic syndrome. Clin Endocrinol (Oxf) 2007;67 (4):479-484

52 Huang-Doran I, Sleigh A, Rochford JJ, O'Rahilly S, Savage DB. Lipodystrophy: metabolic insights from a rare disorder. J Endocrinol 2010;207(3):245-255

53 Javor ED, Ghany MG, Cochran EK, et al. Leptin reverses nonalcoholic steatohepatitis in patients with severe lipodystrophy. Hepatology 2005;41(4):753-760

54 Lüdtke A, Genschel J, Brabant G, et al. Hepatic steatosis in Dunnigan-type familial partial lipodystrophy. Am J Gastroenterol 2005; 100(10):2218-2224

55 Powell EE, Searle J, Mortimer R. Steatohepatitis associated with limb lipodystrophy. Gastroenterology 1989;97(4):10221024

56 Harbour JR, Rosenthal P, Smuckler EA. Ultrastructural abnormalities of the liver in total lipodystrophy. Hum Pathol 1981;12 (9):856-862

57 de Craemer D, Van Maldergem L, Roels F. Hepatic ultrastructure in congenital total lipodystrophy with special reference to peroxisomes. Ultrastruct Pathol 1992;16(3):307-316

58 Bodasing N, Fox R. HIV-associated lipodystrophy syndrome: description and pathogenesis. J Infect 2003;46(3):149-154

59 Dolce CJ, Russo M, Keller JE, et al. Does liver appearance predict histopathologic findings: prospective analysis of routine liver biopsies during bariatric surgery. Surg Obes Relat Dis 2009;5(3): 323-328

60 Abrams GA, Kunde SS, Lazenby AJ, Clements RH. Portal fibrosis and hepatic steatosis in morbidly obese subjects: a spectrum of nonalcoholic fatty liver disease. Hepatology 2004;40(2):475483

61 Kallwitz ER, Guzman G, TenCate V, et al. The histologic spectrum of liver disease in African-American, non-Hispanic white, and Hispanic obesity surgery patients. Am J Gastroenterol 2009;104(1): 64-69

62 Merriman RB, Ferrell LD, Patti MG, et al. Correlation of paired liver biopsies in morbidly obese patients with suspected nonalcoholic fatty liver disease. Hepatology 2006;44(4):874-880 